\title{
Directional Evolution of the Ruled Surfaces via the Evolution of Their Directrix Using q-frame along a Timelike Space Curve
}

\author{
Gül Uğur Kaymanlı ${ }^{1 *}$, Cumali Ekici ${ }^{2}$, Mustafa Dede ${ }^{3}$

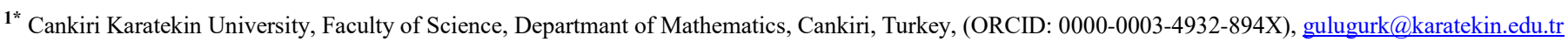 \\ ${ }^{2}$ Eskisehir Osmangazi University, Faculty of Science, Departmant of Mathematics and Computer Science, Eskisehir, Turkey, (ORCID: 0000-0002-3247-5727), \\ cekici@ogu.edu.tr \\ ${ }^{3}$ Kilis 7 Aralik University, Faculty of Science, Departmant of Mathematics, Kilis, Turkey, (ORCID: 0000-0003-2652-637X), mustafadede@kilis.edu.tr
}

(First received 29 Ocak 2020 and in final form 19 Ekim 2020)

(DOI: $10.31590 /$ ejosat.681674)

ATIF/REFERENCE: Kaymanl1, G. U., Ekici, C. \& Dede, M. (2020). Directional Evolution of the Ruled Surfaces via the Evolution of Their Directrix Using q-frame along a Timelike Space Curve. European Journal of Science and Technology, (20), $392-396$.

\begin{abstract}
In this study, the ruled surfaces obtained by normal and binormal vectors along a timelike space curve by using q-frame are investigated in 3 dimensional Minkowski space. Directional evolutions of both quasi normal and quasi binormal ruled surfaces are studied by using their directrices. Then, we work on some geometric properties such as inextensibilty, developability and minimality of these ruled surfaces.
\end{abstract}

Keywords: Evolution surface, Minkowski space, q-frame, Ruled surface.

\section{Timelike Uzay Eğrisi Boyunca q-çatı Kullanılarak Doğrultmanların Gelişimine Göre Regle Yüzeylerin Yönlü Gelişimleri}

$\ddot{O} z$

Bu çalışmada, 3 boyutlu Minkowski uzayında q-çatılı timelike uzay eğrisi boyunca normal ve binormal vektörlerle elde edilen regle yüzeyler incelendi. Doğrultmanlar kullanılarak hem quasi normal hem de quasi binormal regle yüzeylerin yönlü gelişimleri çalışıldı. Daha sonra bu regle yüzeylerin açılabilirliği, uzatılamaz olduğu ve minimal olma özellikleri gibi bazı geometrik özellikler üzerine çalıştık.

Anahtar Kelimeler: Gelişim Yüzeyi, Minkowski Uzayı, q-çatı, Regle Yüzeyi.

\footnotetext{
Sorumlu Yazar: Cankiri Karatekin University, Department of Mathematics, Cankiri, Turkey, ORCID: 0000-0003-4932-894X, gulugurk@karatekin.edu.tr
} 


\section{Introduction}

The time evolution of a curve or a surface is generated by inextensible flows of a curve or a surface. The flow of a curve or a surface is said to be inextensible if its arclength is preserved or the intrinsic curvature is preserved, respectively. Physically, the inextensible curve flows lead to motions in which no strain energy is induced. Also, the evolutions of curves have many important applications of physics as magnetic spin chains and vortex filaments $[3,12,17]$.

In recent times, the motion of inelastic plane curves has been studied by many authors. After Da Rios in 1906 found out the geometric relation between the motion of curves and the differential equation, Doliwa in 1994 [9] characterized the integrable motions of a curve. While Kwon 2005 [16] and Körpinar 2011 [15] worked on inextensible flows of curves in Euclidean space, Gurbuz 2009 [11] and Yüzbaş 2018 [22] studied these curves in Minkowski space. Abd. Ellah 2015, Hussein 2016, D.W. Yoon 2019, Soliman 2018 studied the evolutions of the ruled surfaces via the evolution of their directrix $[1,13,20,21]$. After the quasi-normal vector of a curve was introduced by Coquillart [5], Dede et.al. [6] found quasi frame in 2015 and Soliman also used this frame to work on this subject in 2018 [20].

For a space curve $\alpha(t)$, quasi frame consists of three orthonormal vectors, the unit tangent vector $\mathbf{t}$, the quasi-normal $\mathbf{n}_{q}$ and the quasi-binormal vector $\mathbf{b}_{q}$. The quasi frame as called q-frame $\left\{\mathbf{t}, \mathbf{n}_{q}, \mathbf{b}_{q}\right\}$ is written as

$$
\mathbf{t}=\frac{\alpha^{\prime}}{\left\|\alpha^{\prime}\right\|}, \mathbf{n}_{q}=\frac{\mathbf{t} \wedge \mathbf{k}}{\|\mathbf{t} \wedge \mathbf{k}\|}, \mathbf{b}_{q}=\mathbf{t} \wedge \mathbf{n}_{q},
$$

where $\mathbf{k}$ is the projection vector [6]. The q-frame has many advantages versus other frames (Frenet, Bishop). For instance the q-frame can be defined even along a line $(\kappa=0)$ and the construction of the q-frame doesn't change if the space curve has unit speed or not and the q-frame can also be calculated easily [6].

The projection vector $\mathbf{k}$ is a unit vector along $x-, y-$ and $z-$ axes. We choose the projection vector $\mathbf{k}=(0,0,1)$ without loss of generality. A quasi frame along a space curve is shown in Figure 1.

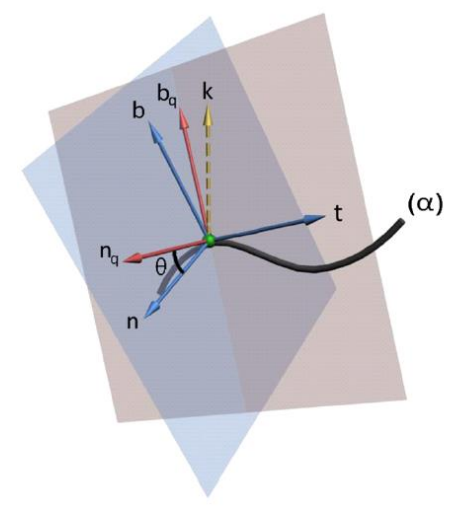

Figure 1: The q-frame and Frenet frame.

The derivation equations of the quasi frame is expressed by

$$
\left[\begin{array}{c}
\mathbf{t}^{\prime} \\
\mathbf{n}_{q}^{\prime} \\
\mathbf{b}_{q}^{\prime}
\end{array}\right]=\left\|\alpha^{\prime}\right\|\left[\begin{array}{ccc}
0 & k_{1} & k_{2} \\
-k_{1} & 0 & k_{3} \\
-k_{2} & -k_{3} & 0
\end{array}\right]\left[\begin{array}{c}
\mathbf{t} \\
\mathbf{n}_{q} \\
\mathbf{b}_{q}
\end{array}\right],
$$

where the quasi curvatures are given as follows

$$
k_{1}=-\frac{\left\langle\mathbf{t}, \mathbf{n}_{q}^{\prime}\right\rangle}{\left\|\alpha^{\prime}\right\|}, k_{2}=-\frac{\left\langle\mathbf{t}, \mathbf{b}_{q}^{\prime}\right\rangle}{\left\|\alpha^{\prime}\right\|}, k_{3}=\frac{\left\langle\mathbf{n}_{q}^{\prime}, \mathbf{b}_{q}\right\rangle}{\left\|\alpha^{\prime}\right\|} .
$$

A ruled surface is a surface that can be sweept out by moving a line in space. Therefore, it has a parametrization of the form

$$
\varphi(u, v)=\alpha(u)+v \delta(u)
$$

where $\alpha$ is called the directrix and $\delta$ is the director curve.

In Minkowski 3-space $\mathbb{R}_{1}^{3}$, the inner product of two vectors $\mathbf{u}=\left(u_{1}, u_{2}, u_{3}\right)$ and $\mathbf{w}=\left(w_{1}, w_{2}, w_{3}\right)$ is defined as

$$
<\mathbf{u}, \mathbf{w}>=u_{1} w_{1}+u_{2} w_{2}-u_{3} w_{3}
$$

and the cross product of two vectors $\mathbf{u}=\left(u_{1}, u_{2}, u_{3}\right)$ and $\mathbf{w}=\left(w_{1}, w_{2}, w_{3}\right)$ is defined as

$$
\mathbf{u} \wedge \mathbf{w}=\left(u_{3} w_{2}-u_{2} w_{3}, u_{1} w_{3}-u_{3} w_{1}, u_{1} w_{2}-u_{2} w_{1}\right)
$$

where $\mathbf{e}_{1} \wedge \mathbf{e}_{2}=\mathbf{e}_{3}, \quad \mathbf{e}_{2} \wedge \mathbf{e}_{3}=-\mathbf{e}_{1}, \quad \mathbf{e}_{3} \wedge \mathbf{e}_{1}=-\mathbf{e}_{2}$, respectively [2]. If $\mathbf{u}$ and $\mathbf{w}$ are timelike vectors then

$\mathbf{u} \wedge \mathbf{W}$ is a spacelike vector [19].

The norm of the vector $\mathbf{W}$ is given by

$$
\|\mathbf{w}\|=\sqrt{|\langle\mathbf{w}, \mathbf{w}\rangle|}
$$

We say that a Lorentzian vector $\mathbf{w}$ is spacelike, lightlike or timelike if $\langle\mathbf{w}, \mathbf{w}\rangle>0,\langle\mathbf{w}, \mathbf{w}\rangle=0$ and $\mathbf{w} \neq 0$, $\langle\mathbf{w}, \mathbf{w}\rangle<0$, respectively. In particular, the vector $\mathbf{w}=0$ is spacelike [18,19]. Let $\alpha(t)$ be a timelike space curve with a non-vanishing second derivative.

Then Frenet formulas of timelike curve may be written as

$$
\left[\begin{array}{c}
\mathbf{t}^{\prime} \\
\mathbf{n}^{\prime} \\
\mathbf{b}^{\prime}
\end{array}\right]=v\left[\begin{array}{ccc}
0 & \kappa & 0 \\
\kappa & 0 & \tau \\
0 & -\tau & 0
\end{array}\right]\left[\begin{array}{l}
\mathbf{t} \\
\mathbf{n} \\
\mathbf{b}
\end{array}\right]
$$

where $v=\|\mathbf{t}\|$. The curvature and torsion of timelike curve $\alpha(t)$ in Minkowski 3-space are obtained by

$$
\kappa=\left\|\mathbf{t}^{\prime}\right\|, \tau=<\mathbf{n}^{\prime}, \mathbf{b}>
$$

respectively $[2,19]$

Let $\varphi$ be a surface in Euclidean 3-space, the first fundamental form $I$ of $\varphi$ is defined by $I=E d s^{2}+2 F d s d v+G d v^{2}$ where

$$
E=<\varphi_{s}, \varphi_{s}>, F=<\varphi_{s}, \varphi_{v}>, G=<\varphi_{v}, \varphi_{v}>.
$$

The second fundamental form II of $\varphi$ is given by $I I=e d s^{2}+2 f d s d v+g d v^{2}$ where

$$
e=<\varphi_{s s}, N>, f=<\varphi_{s v}, N>, g=<\varphi_{v v}, N>
$$

and $N$ is the unit normal of $\varphi$. The Gaussian and the mean curvatures are expressed as 


$$
K=\frac{e g-f^{2}}{E G-F^{2}} \text { and } H=\frac{e G-2 f F+g E}{2\left(E G-F^{2}\right)}
$$

respectively. The necessary and sufficient condition for a regular surface being developable and flat is that its Gaussian curvature vanishes identically. A minimal surface in $\mathrm{R}^{\mathbf{3}}$ is a regular surface if its mean curvature vanishes identically [4].

Moreover, in [22] a surface evolution $\varphi(s, v, t)$ and its flow $\frac{\partial \varphi}{\partial t}$ are said to be inextensible if the following equalities satisfy

$$
\frac{\partial E}{\partial t}=\frac{\partial F}{\partial t}=\frac{\partial G}{\partial t}=0
$$

In this paper, we give another approach to evolutions of the ruled surfaces depend on a timelike space curve by q-frame used in $[7,8,10,14]$. Using $\mathrm{q}$-frame, we present two sets of quasi frame equations with respect to arc-length $S$ and time $t$. We obtain three differential equations depend on q-curvatures for the qframe vectors of the timelike space curve. Calculating first and second fundamental forms of this ruled surface, we get geometric properties such as curvatures, flatness, inextensibility and minimality of the ruled surface.

\section{2. q-frame Along a Timelike Space Curve}

As an alternative frame, quasi frame as called $\mathrm{q}$-frame in both Euclidean and Minkowski space is defined by Dede and Ekici et al. [6,10]. For a space curve $\alpha(t)$, quasi frame consists of three orthonormal vectors, the unit tangent vector $\mathbf{t}$, the quasi-normal $\mathbf{n}_{q}$ and the quasi-binormal vector $\mathbf{b}_{q}$. The quasi frame as called q-frame $\left\{\mathbf{t}, \mathbf{n}_{q}, \mathbf{b}_{q}\right\}$ is written as

$$
\mathbf{t}=\frac{\alpha^{\prime}}{\left\|\alpha^{\prime}\right\|}, \mathbf{n}_{q}=\frac{\mathbf{t} \wedge \mathbf{k}}{\|\mathbf{t} \wedge \mathbf{k}\|}, \mathbf{b}_{q}=\mathbf{t} \wedge \mathbf{n}_{q} .
$$

Since the derivation formula for the q-frame for the timelike curve in Minkowski space does not depend on projection vector being timelike or spacelike, we work on spacelike projection vector without loss of generality.

In [8], the derivation equations of the directional q-frame for the timelike space curve when tangent vector (timelike), projection vector $\mathbf{k}=(0,1,0) \quad$ (spacelike), quasi-normal vector (spacelike) and quasi-binormal vector (spacelike) are given by

$$
\left[\begin{array}{c}
\mathbf{t}^{\prime} \\
\mathbf{n}_{q}^{\prime} \\
\mathbf{b}_{q}^{\prime}
\end{array}\right]=\left[\begin{array}{ccc}
0 & k_{1} & k_{2} \\
k_{1} & 0 & k_{3} \\
k_{2} & -k_{3} & 0
\end{array}\right]\left[\begin{array}{l}
\mathbf{t} \\
\mathbf{n}_{q} \\
\mathbf{b}_{q}
\end{array}\right],
$$

where the q-curvatures are

$$
k_{1}=\left\langle\mathbf{t}^{\prime}, \mathbf{n}_{q}\right\rangle, k_{2}=\left\langle\mathbf{t}^{\prime}, \mathbf{b}_{q}\right\rangle, k_{3}=\left\langle\mathbf{n}_{q}^{\prime}, \mathbf{b}_{q}\right\rangle
$$

Then, we have a relation matrix in the following form

$$
\left[\begin{array}{l}
\mathbf{t} \\
\mathbf{n}_{q} \\
\mathbf{b}_{q}
\end{array}\right]=\left[\begin{array}{ccc}
1 & 0 & 0 \\
0 & \cosh \theta & \sinh \theta \\
0 & -\sinh \theta & -\cosh \theta
\end{array}\right]\left[\begin{array}{l}
\mathbf{t} \\
\mathbf{n} \\
\mathbf{b}
\end{array}\right] .
$$

Thus,

$$
\left[\begin{array}{l}
\mathbf{t} \\
\mathbf{n} \\
\mathbf{b}
\end{array}\right]=\left[\begin{array}{ccc}
1 & 0 & 0 \\
0 & \cosh \theta & \sinh \theta \\
0 & -\sinh \theta & -\cosh \theta
\end{array}\right]\left[\begin{array}{l}
\mathbf{t} \\
\mathbf{n}_{q} \\
\mathbf{b}_{q}
\end{array}\right] .
$$

Moreover, the relation between q-curvatures and Frenet curvatures are as follows

$$
k_{1}=\kappa \cos \theta, \quad k_{2}=-\kappa \sin \theta, \quad k_{3}=d \theta+\tau .
$$

\section{Evolution of Timelike Space Curve with Time by q-frame}

In this section, we obtain time evolution equations depending on q-curvatures of the evolving curve in order to obtain space curve with $\mathrm{q}$-frame. That is, integrating time evolution equations for given $\lambda, \mu, \eta$, one can find $\mathrm{q}$ curvatures. Using eq. (6), we get evolving curve.

Theorem 1. The evolution equations for the quasi curvatures of the evolving curve are given by

$$
\begin{aligned}
& \frac{\partial k_{1}}{\partial t}=k_{2} \eta-k_{3} \mu+\frac{\partial \lambda}{\partial s} \\
& \frac{\partial k_{2}}{\partial t}=k_{3} \lambda-k_{1} \eta+\frac{\partial \mu}{\partial s} \\
& \frac{\partial k_{3}}{\partial t}=k_{2} \lambda-k_{1} \mu+\frac{\partial \eta}{\partial s}
\end{aligned}
$$

where the quasi formula with respect to time $t$ is in the form

$$
\frac{\partial}{\partial t}\left[\begin{array}{c}
\mathbf{t} \\
\mathbf{n}_{q} \\
\mathbf{b}_{q}
\end{array}\right]=\left[\begin{array}{ccc}
0 & \lambda & \mu \\
\lambda & 0 & \eta \\
\mu & -\eta & 0
\end{array}\right]\left[\begin{array}{c}
\mathbf{t} \\
\mathbf{n}_{q} \\
\mathbf{b}_{q}
\end{array}\right]
$$

Proof. Using equation (11), and defining

$$
q=\left[\begin{array}{c}
\mathbf{t} \\
\mathbf{n}_{q} \\
\mathbf{b}_{q}
\end{array}\right], A=\left[\begin{array}{ccc}
0 & k_{1} & k_{2} \\
k_{1} & 0 & k_{3} \\
k_{2} & -k_{3} & 0
\end{array}\right], B=\left[\begin{array}{ccc}
0 & \lambda & \mu \\
\lambda & 0 & \eta \\
\mu & -\eta & 0
\end{array}\right],
$$

we can write

$$
\begin{aligned}
& \frac{\partial q}{\partial s}=A q, \\
& \frac{\partial q}{\partial t}=B q .
\end{aligned}
$$

Applying the compatibility condition

$$
\frac{\partial}{\partial t}\left(\frac{\partial q}{\partial s}\right)=\frac{\partial}{\partial s}\left(\frac{\partial q}{\partial t}\right)
$$

one can find easily

$$
\frac{\partial A}{\partial t}-\frac{\partial B}{\partial s}+[A, B]=0
$$


where $[A, B]=A B-B A$ is called Lie bracket of $A$ and $B$.

Using the equations (12) and (14), the matrix of evolution equations is obtained by

$\left[\begin{array}{ccc}0 & \frac{\partial k_{1}}{\partial t}-\frac{\partial \lambda}{\partial s}-k_{2} \eta+k_{3} \mu & \frac{\partial k_{2}}{\partial t}-\frac{\partial \mu}{\partial s}+k_{1} \eta-k_{3} \lambda \\ \frac{\partial k_{1}}{\partial t}-\frac{\partial \lambda}{\partial s}+k_{3} \mu-k_{2} \eta & 0 & \frac{\partial k_{3}}{\partial t}-\frac{\partial \eta}{\partial s}+k_{1} \mu-k_{2 \lambda} \\ \frac{\partial k_{2}}{\partial t}-\frac{\partial \mu}{\partial s}-k_{3} \lambda+k_{1} \mu & -\frac{\partial k_{3}}{\partial t}+\frac{\partial \eta}{\partial s}+k_{2} \lambda+k_{1} \mu & 0\end{array}\right]=0$

Thus the compatibility condition becomes

which proves theorem.

$$
\begin{aligned}
& \frac{\partial k_{1}}{\partial t}=k_{2} \eta-k_{3} \mu+\frac{\partial \lambda}{\partial s} \\
& \frac{\partial k_{2}}{\partial t}=k_{3} \lambda-k_{1} \eta+\frac{\partial \mu}{\partial s} \\
& \frac{\partial k_{3}}{\partial t}=k_{2} \lambda-k_{1} \mu+\frac{\partial \eta}{\partial s}
\end{aligned}
$$

\section{Directional Evolutions of the Ruled Surfaces Depending on A Timelike Space Curve}

In this section, giving the characterizations of evolutions of ruled surfaces generated by both quasi normal and quasi binormal, we calculate their Gaussian and mean curvatures.

\subsection{Evolution of Quasi Normal Ruled Surface}

The equation of surfaces generated by quasi normal $\mathrm{i}$

$$
\varphi(s, v, t)=\alpha(s, t)+v \mathbf{n}_{q}(s, t)
$$

First partial derivatives of the surface $\varphi(s, v, t)$ are

$$
\varphi_{s}=\left(1+v k_{1}\right) \mathbf{t}+v k_{3} \mathbf{b}_{q} \text { and } \varphi_{v}=\mathbf{n}_{q} .
$$

The normal vector of $\varphi(s, v, t)$ is

$$
N=\frac{\varphi_{s} \wedge \varphi_{v}}{\left\|\varphi_{s} \wedge \varphi_{v}\right\|}=\frac{-v k_{3} \mathbf{t}-\left(1+v k_{1}\right) \mathbf{b}_{q}}{\sqrt{\left|-v^{2} k_{3}^{2}+\left(1+v k_{1}\right)^{2}\right|}}
$$

The coefficients of the first fundamental form are calculated by

$$
\begin{aligned}
& E=v^{2}\left(k_{3}^{2}-k_{1}^{2}\right)-2 v k_{1}-1 \\
& F=0 \\
& G=1 .
\end{aligned}
$$

Second partial derivatives of the surface $\varphi(s, v, t)$ are

$$
\begin{aligned}
\varphi_{s s} & =v\left(\frac{\partial k_{1}}{\partial s}+k_{2} k_{3}\right) \mathbf{t}+\left(\left(1+v k_{1}\right) k_{1}-v k_{3}^{2}\right) \mathbf{n}_{q}+\left(\left(1+v k_{1}\right) k_{2}+v \frac{\partial k_{3}}{\partial s}\right) \mathbf{b}_{q} \\
\varphi_{s v} & =k_{1} \mathbf{t}+k_{3} \mathbf{b}_{q} \\
\varphi_{v v} & =\frac{\partial \mathbf{n}_{q}}{\partial v}=0 .
\end{aligned}
$$

The coefficients of the second fundamental form are calculated by

$$
e=\frac{-k_{2}-v\left(2 k_{1} k_{2}-\frac{\partial k_{3}}{\partial s}\right)+v^{2}\left[k_{3}^{2} \frac{\partial}{\partial s}\left(\frac{k_{1}}{k_{3}}\right)+k_{2}\left(k_{3}^{2}-k_{1}^{2}\right)\right]}{\sqrt{\left|1+v^{2}\left(k_{1}^{2}-k_{3}^{2}\right)+2 v k_{1}\right|}}
$$$$
f=\frac{v k_{1}\left(1-k_{3}\right)-k_{3}}{\sqrt{\left|1+v^{2}\left(k_{1}^{2}-k_{3}^{2}\right)+2 v k_{1}\right|}}
$$$$
g=0 \text {. }
$$

One can find Gaussian and mean curvatures

$$
K=-\frac{f^{2}}{E}=\frac{-k_{3}^{2}+2 v k_{1} k_{3}\left(1-k_{3}\right)-v^{2} k_{1}^{2}\left(1-2 k_{3}+k_{3}^{2}\right)}{\left(1+v^{2}\left(k_{1}^{2}-k_{3}^{2}\right)+2 v k_{1}\right)^{2} \cdot\left(v^{2}\left(k_{3}^{2}-k_{1}^{2}\right)-2 v k_{1}-1\right)}
$$

and

$$
H=\frac{e}{2 E}=\frac{-k_{2}-v\left(2 k_{1} k_{2}-\frac{\partial k_{3}}{\partial s}\right)+v^{2}\left[k_{3}^{2} \frac{\partial}{\partial s}\left(\frac{k_{1}}{k_{3}}\right)+k_{2}\left(k_{3}^{2}-k_{1}^{2}\right)\right]}{2\left(1+v^{2}\left(k_{1}^{2}-k_{3}^{2}\right)+2 v k_{1}\right)^{3 / 2}}
$$

respectively. The quasi normal ruled surface is minimal if and only if $e=0$.

The ruled surfaces generated by quasi normal are developable and flat if and only if

$$
k_{3}=\frac{1}{1+\left(v k_{1}\right)^{-1}} .
$$

With the help of (10), if the quasi normal surface is inextensible, then one can derive this differential equation

$$
v^{2} \frac{\partial}{\partial t}\left(k_{3}^{2}-k_{1}^{2}\right)-v \frac{\partial k_{1}}{\partial t}=0 \text {. }
$$

\subsection{Evolution of Quasi Binormal Ruled Surface}

The equation of surfaces generated by quasi binormal is

$$
\varphi(s, v, t)=\alpha(s, t)+v \mathbf{b}_{q}(s, t)
$$

First partial derivatives of the surface $\varphi(s, v, t)$ are

$$
\begin{aligned}
\varphi_{s} & =\left(1+v k_{2}\right) \mathbf{t}-v k_{3} \mathbf{n}_{q} \\
\varphi_{v} & =\mathbf{b}_{q} .
\end{aligned}
$$

The normal vector of $\varphi(s, v, t)$ is

$$
N=\frac{\varphi_{s} \wedge \varphi_{v}}{\left\|\varphi_{s} \wedge \varphi_{v}\right\|}=\frac{-v k_{3} \mathbf{t}+\left(1+v k_{2}\right) \mathbf{n}_{q}}{\sqrt{\left|1-v^{2} k_{3}^{2}+\left(1+v k_{2}\right)^{2}\right|}} .
$$

The coefficients of the first fundamental form are calculated by

$$
\begin{aligned}
& E=v^{2}\left(k_{3}^{2}-k_{2}^{2}\right)-2 v k_{2}-1 \\
& F=0 \\
& G=1 .
\end{aligned}
$$

Second partial derivatives of the surface $\varphi(s, v, t)$ are 
$\varphi_{s s}=v\left(\frac{\partial k_{2}}{\partial s}-k_{1} k_{3}\right) \mathbf{t}+\left(k_{1}+v k_{1} k_{2}-v \frac{\partial k_{3}}{\partial s}\right) \mathbf{n}_{q}+\left(k_{2}+v k_{2}^{2}-v k_{3}^{2}\right) \mathbf{b}_{q}$

$\varphi_{s v}=k_{2} \mathbf{t}-k_{3} \mathbf{n}_{q}$

$\varphi_{v v}=0$.

The coefficients of the second fundamental form are calculated by

$$
\begin{aligned}
& e=\frac{k_{1}+v\left(2 k_{1} k_{2}-\frac{\partial k_{3}}{\partial s}\right)+v^{2}\left[k_{3}^{2} \frac{\partial}{\partial s}\left(\frac{k_{2}}{k_{3}}\right)+k_{1}\left(k_{2}^{2}-k_{3}^{2}\right)\right]}{\sqrt{\left|1+v^{2}\left(k_{2}^{2}-k_{3}^{2}\right)+2 v k_{2}\right|}} \\
& f=\frac{-k_{3}}{\sqrt{\left|1-v^{2} k_{3}^{2}+\left(1+v k_{2}\right)^{2}\right|}} \\
& g=0 .
\end{aligned}
$$

Gaussian and mean curvatures are calculated by

$$
K=\left(\frac{k_{3}}{1-v^{2} k_{3}^{2}+\left(1+v k_{2}\right)^{2}}\right)^{2}
$$

and

$$
H=\frac{k_{1}+v\left(2 k_{1} k_{2}-\frac{\partial k_{3}}{\partial s}\right)+v^{2}\left[k_{3}^{2} \frac{\partial}{\partial s}\left(\frac{k_{2}}{k_{3}}\right)+k_{1}\left(k_{2}^{2}-k_{3}^{2}\right)\right]}{2\left(1-v^{2} k_{3}^{2}+\left(1+v k_{2}\right)^{2}\right)^{3 / 2}}
$$

respectively. The quasi binormal ruled surface is minimal if and only if $e=0$.

The ruled surfaces generated by quasi binormal are both developable and flat if and only if $k_{3}=0$. With the help of (10), if the quasi binormal surface is inextensible, then one can derive this differential equation

$$
v^{2} \frac{\partial}{\partial t}\left(k_{3}^{2}-k_{2}^{2}\right)-v \frac{\partial k_{2}}{\partial t}=0
$$

\section{References}

[1] Abd-Ellah, H. N. (2015) Evolution of translation Surfaces in Euclidean 3 space $\mathbf{E}^{3}$, Applied Mathematics and Information Science. 9(2), 661-668.

[2] Akutagawa, K., and Nishikawa, S. (1990) The Gauss map and spacelike surfaces with prescribed mean curvature in Minkowski 3-space, Tohoku Math. J. 42(2): 67-82.
[3] Balakrishnan, R., Blumenfeld, R. (1997) Transformation of general curve evolution to a modified Belavin-Polyakov equation. J. Math. Phys., 38(11), 5878-5888.

[4] Carmo, M. (1976) Differential Geometry of Curves and Surfaces, Prentice-Hall, Saddle River.

[5] Coquillart, S. (1987) Computing offsets of B-spline curves, Computer-Aided Design, 19(6): 305-09.

[6] Dede, M., Ekici, C., and Gorgulu, A. (2015) Directional qframe along a space curve, IJARCSSE. 5(12), 775-780.

[7] Dede, M., Ekici, C., and Tozak, H. (2015) Directional tubular surfaces, International Journal of Algebra. 9(12), 527-535.

[8] Dede, M., Tarım, G., and Ekici, C. (2017) Timelike directional Bertrand curves in Minkowski Space, 15th International Geometry Symposium, Amasya, Turkey.

[9] Doliwa, A., and Santini, P. (1994) An elementary geometric characterisation of the integrable motions of a curve. Physics Letters A, 185, 373-384.

[10] Ekici, C., Dede, M., Tozak, H. (2017) Timelike directional tubular surfaces, Int. J. Mathematical Anal., 8(5), 1-11.

[11] Gurbuz, N. (2009) Inextensible flows of spacelike, timelike and null curves. Int. J. Contemp Math Sci., 4, 1599-1604.

[12] Hasimoto, H. (1959) A soliton on a vortex filament. J. Fluid Mech., 51(3), 477-485.

[13] Hussien, R.A., and Youssef, T. (2016) Evolution of Special Ruled Surfaces via the Evolution of Their Directrices in Euclidean 3-Space $\mathbf{E}^{\mathbf{3}}$, Applied Mathematics and Information Science. 10(5), 1949-1956.

[14] Kaymanli, G.U., Ekici, C. and Dede, M. (2018) Directional canal surfaces in E3, Current Academic Studies in Natural Sciences and Mathematics Sciences, 63-80.

[15] Korpinar, T., Asil, V. and Baş, S. (2011) On characterization inextensible flows of curves according to Bishop frame, Notas de Matematica, 7(1), 37-45.

[16] Kwon, D.Y., Park, F.C. (2005) Inextensible flows of curves and developable surfaces, Appl. Math. Lett., 18, 1156-1162.

[17] Lakshmanan, M., Ruijgrok, T.W., Thompson, C.J. (1976) On the dynamics of a continuum spin system, Phys. A, 84(3), 577-590.

[18] Lopez, R. (2014) Differential geometry of curves and surfaces in Lorentz-Minkowski space. International Electronic Journal of Geometry, 7, 44-107.

[19] O’Neill, B. (1983) Semi-Riemannian geometry with applications to relativity. Academic Press, New York.

[20] Soliman, M.A., Abdel-All, N.H., Hussein, R.A. and Youssef, T. (2018) Evolutions of the Ruled Surface via the Evolution of Their Directrix Using Quasi Frame along a Space Curve, Journal of Applied Mathematics and Physics. 6, 1748-1756.

[21] Yoon, D.W., Yuzbasi, Z.K. and Aslan, E.C. (2019) Evolution of Spacelike Curves and Special Timelike Ruled Surfaces in the Minkowski Space, arXiv:1908.00053v1.

[22] Yuzbasi, Z.K., Yoon, D.W. (2018) Inextensible Flows of Curves on Lightlike Surfaces. Mathematics, 6(11), 224. 